$$
\begin{aligned}
& \text { Pontifícia Universidade Católica } \\
& \text { DO RIO DE JANEIRO }
\end{aligned}
$$

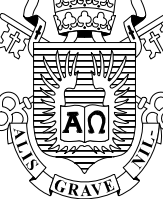

Mariana de Souza Guimarães

\title{
O design dos objetos artesanais produzidos no cotidiano de mulheres idosas
}

\section{Dissertação de Mestrado}

Dissertação apresentada ao Programa de Pós-graduação em Design do Departamento de Artes \& Design da PUC-Rio como parte dos requisitos parciais para obtenção do título de Mestre em Design.

Orientador: Prof. Alberto Cipiniuk 


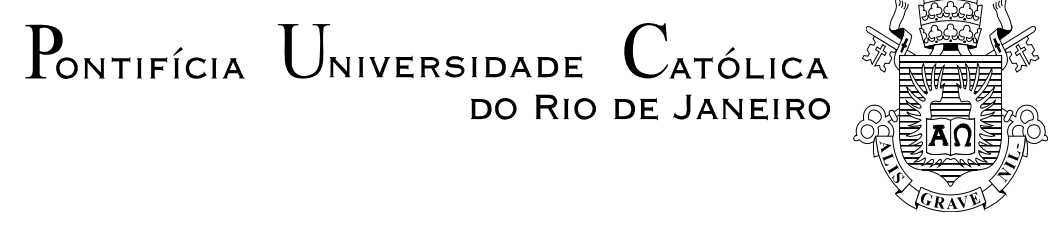

\title{
Mariana de Souza Guimarães
}

\section{O design dos objetos artesanais produzidos no cotidiano de mulheres idosas}

\begin{abstract}
Dissertação apresentada ao Programa de Pós-Graduação em Design da PUC-Rio como requisito parcial para obtenção do título de Mestre em Design. Aprovada pela Comissão Examinadora abaixo assinada.
\end{abstract}

Prof. Alberto Cipiniuk Orientador Departamento de Artes \& Design - PUC-Rio

Profa. Doris Clara Kosminsky Co-orientador UFRJ

Profa. Carla da Costa Dias UFRJ

Profa. Rita Maria de Souza Couto Departamento de Artes \& Design - PUC-Rio

Prof. Paulo Fernando Carneiro de Andrade Coordenador Setorial do Centro de Teologia e Ciências Humanas - PUC-Rio 
Todos os direitos reservados. É proibida a reprodução total ou parcial do trabalho sem autorização da autora, do orientador e da Universidade.

\section{Mariana de Souza Guimarães}

Graduou-se em Licenciatura em Educação Artística pela Escola de Belas Artes da Universidade Federal do Rio de Janeiro no ano de 2005. é idealizadora e coordenadora do Projeto Retalhos de Memória.

Guimarães, Mariana de Souza

O design dos objetos artesanais produzidos no cotidiano de mulheres idosas / Mariana de Souza Guimarães; orientador: Alberto Cipiniuk; co-orientador: Doris Clara Kosminsky - 2010

$108 \mathrm{f.} \mathrm{;} 30 \mathrm{~cm}$

Dissertação (Mestrado em Design) - Pontifícia Universidade Católica do Rio de Janeiro, Departamento de Artes e Design, 2010.

Inclui bibliografia

1. Artes e Design - Dissertações. 2. Design popular. 3. Bordado. I. Cipinuk, Alberto. II. Doris Clara Kosminsky. III. Pontifícia Universidade Católica do Rio de Janeiro. Departamento de Artes e Design. IV. Título. 
Dedico esse trabalho a todas as participantes do

Projeto Retalhos de Memória, que muito me ensinaram com suas histórias. 


\section{Agradecimentos}

Agradeço à minha espiritualidade, que me faz ter fé nesse trabalho, na vida e no ser humano, pela força e intuição. Aos meus familiares, pelo apoio e carinho. Especialmente, ao meu companheiro, Márcio Macedo, pela paciência e atenção em ouvir minhas histórias e por estar ao meu lado nesta jornada, que é a vida.

Ao Professor Alberto Cipiniuk, pela generosidade, coerência e inquietude qualidades essenciais que nortearam o trabalho da orientação.

Aos demais professores do Programa de Pós-graduação em Artes \& Design, pelas sugestões e observações e, aos funcionários, pela atenção e zelo.

À professora Carla Dias, pelo esclarecimento de tantas questões sobre o universo da arte popular brasileira.

Aos colegas, sobretudo do grupo de estudo GRUDAR, pela amizade, questionamentos e pela coesão nos nossos encontros e ideais.

A CAPES, pela concessão de bolsa de estudo durante todo o curso de mestrado.

Às participantes do Projeto Retalhos de Memória, pela confiança e incentivo ao Projeto Retalhos de Memória. 


\section{Resumo}

Guimarães, Mariana de Souza; Cipiniuk, Alberto Cipiniuk (Orientador); Kosminsky, Doris Clara (Co-orientador). $\mathrm{O}$ design dos objetos artesanais produzidos no cotidiano de mulheres idosas. Rio de Janeiro, 2010. 108p. Dissertação de Mestrado - Departamento de Artes e Design, Pontifícia Universidade Católica do Rio de Janeiro.

Este estudo tem como tema o design de objetos artesanais produzidos por mulheres idosas, com linhas e agulhas. O objetivo é sinalizar os pontos de divergência e convergência entre os campos do design e do artesanato popular, identificando relações híbridas, e compreendendo as fronteiras existentes entre os campos e conhecendo modos distintos de produção de artefatos da cultura material brasileira. Buscamos ainda promover uma reflexão sobre a importância das técnicas aliadas ao ato de costurar, tecer e bordar na história da humanidade, suas origens e eventuais consequências. Técnicas como crochê, tricô, bordado, etc., ligadas essencialmente ao universo feminino, mesmo nos dias atuais, frente à industrialização, não desapareceram. Continuam a existir, em produtos e projetos de design, moda e arte. Por meio de uma pesquisa participativa intitulada Projeto Retalhos de Memória, realizada com aproximadamente 150 mulheres idosas, comprovamos que estas técnicas estão presentes no imaginário e lembranças pessoais das mulheres idosas, pelo fato de terem sido inculcadas e apreendidas na esfera doméstica e institucional pelo habitus e estilo de vida na formação de moças de determinada geração, hoje idosa. A falta de diálogo das gerações mais jovens com as pessoas idosas e a conseqüente exclusão destas da sociedade faz com que muita sabedoria seja perdida na cultura material e imaterial.

\section{Palavras-chave}

Design popular, artesanato, mulher idosa, bordado, memória. 


\section{Abstract}

Guimarães, Mariana de Souza; Cipiniuk, Alberto Cipiniuk (Advisor); Kosminsky, Doris Clara (Co-advisor). The design of handicrafts objects produced in the daily life of elderly womem. Rio de Janeiro, 2010. 108p. MSc Dissertation - Departamento de Artes e Design, Pontifícia Universidade Católica do Rio de Janeiro.

This study deals with the design of handicrafts produced in the daily life of elderly women. Its goal is to establish a discussion between the design and the artifacts produced with needles and lines in the domestic daily life. Signaling points of divergence and convergence between the fields of design and artisan groups, identifying hybrid relationships between them, understanding the boundaries between the fields and getting to know different possibilities for the production of artifacts in the Brazilian material culture. We seek to further the discussion about the importance of the techniques allied to the acts of sewing, weaving and embroidering throughout the history of mankind, its origins and eventual consequences. Techniques such as crochet, knitting, embroidery, etc..., essentially linked to the feminine universe that today, facing industrialization, have not disappeared and are present in products and design projects, fashion and art. Through a participatory research named Projeto Retalhos de Memória, performed with approximately 150 elderly women, we could verify that these techniques are present in the imaginary and personal memories of elderly women because these techniques have been learned and lived in the domestic sphere and institutional environment through the habitus and lifestyle that was present in the education of girls of a certain generation, today elder. The lack of dialogue and the exclusion of older people of our society cause wisdom to be lost in our material and immaterial culture.

\section{Keywords}

Popular design, handicrafts, elder woman, embroidery, memory. 


\section{Sumário}

$\begin{array}{ll}\text { Introdução } & 12\end{array}$

1. Design, artesanato e sociedade contemporânea 18

1. 1. Design, métodos e metodologias $\quad 18$

1.2. Artesanato e sociedade contemporânea 26

1.3. Design e artesanato $\quad 31$

1.4. Design, sociedade industrial e alienação 35

2. Fio-a- fio 41

2.1. Linhas e Agulhas: tempo e espaço 41

2.2. Brasil e técnicas artesanais têxteis 46

2.3. Novos usos: a revolução industrial 49

2.4. Apropriação das técnicas artesanais pela indústria 52

2.5. Iniciativas Híbridas: alguns exemplos 55

2.6. Consumo nos armarinhos: cultura de resistência ou falta de opção? 61

3. Estudo de Campo 66

3.1. Panorama atual e breve histórico do envelhecimento no Brasil 66

3.1.1. Feminização na velhice 69

3.1.2. Envelhecimento e sociedade industrial $\quad 71$

$\begin{array}{ll}\text { 3.2. Habitus e mulher idosa } & 73\end{array}$

3.3. Estudo de caso: Projeto Retalhos de Memória 81

4. Conclusão 102

5. Referências bibliográficas 106 


\section{Índice de imagens}

As imagens reproduzidas neste estudo foram extraídas pela autora em meios eletrônicos, exceto as fotografias reproduzidos no capítulo 03 que foram todas registradas pela autora durante a execução do trabalho de campo intitulado Projeto Retalhos de Memória com as mulheres idosas.

01: Detalhe das mãos de uma mulher nordestina fazendo renda.

Catálogo:As rendeiras do Morro de Mariana - Sebrae - Piauí/ PI.

02: As Môiras, com o fio da vida.

Strudwick, 1885 - www..wikipedia.org

03 : Jovens na aula de costura - Escola Senai, 1949.

Acervo Projeto Memória - Senai/ S.P. In: Maleronka, Wanda.

Fazer Roupa virou moda. Ed: Senac - S.P - pg:150.

04: Artesãs na sede da Coopa Roca.

www.cooparoca.com.br

05: Luminária projetada por Tord Boontje

www.cooparoca.com.br

06: Desfile Apoena - fashion Rio 2010.

www.apoena.com.br

07: Detalhe da Ilustração bordada pela família Dumont.

In: Colasanti, Marina. A moça tecelã.Ed: Global- S.P.

08: Fotografia Armarinho Santa Izabel .Registro da autora. 
09: Gráfico IBGE. www.ibge.gov.br

10: Recorte publicitário. in: A arte de bordar Ed: S. A O malho. s/ data.

11: Recorte publicitário. in : A arte de bordar Ed: S.A O malho s/data.

12: Desenho de participante do Projeto Retalhos de Memória.

13: Fotografia trabalho em fuxico, 2006.

14: Fotografia participante costurando, 2006.

15: Fotografia detalhe do painel de fuxico,2006.

16: Fotografia de participantes costurando e bordando, 2006.

17: Fotografia de participante bordando tecido com sua imagem, 2009.

18: Fotografia idosa observando seu retrato,2009.

19: Fotografia de idosa e seu trabalho,2009.

20: Fotografia de idosa e seu trabalho,2009.

21: Fotografia de idosa bordado sobre sua imagem,2009.

22: Fotografia de idosa observando seu retrato, 2009.

23: Fotografia do trabalho de participante, 2009.

24: Trabalho de participante, 2009.

25: Exposição Retalhos de Memória. Rio de Janeiro, 2009. 
26: Exposição Retalhos de Memória. Rio de Janeiro, 2009.

27: Exposição Retalhos de Memória. Rio de Janeiro, 2009.

28. Painel de fuxico - Exposição Retalhos de Memória. Resende, 2007. 